\title{
Estimates of $L_{p}$ Modulus of Continuity of Generalized Bounded Variation Classes
}

\author{
Heping Wang ${ }^{1}$ and Zhaoyang $\mathrm{Wu}^{2}$ \\ ${ }^{1}$ School of Mathematical Sciences, BCMIIS, Capital Normal University, Beijing 100048, China \\ ${ }^{2}$ NO. 2 Middle School of Changping, Beijing 102208, China
}

Correspondence should be addressed to Heping Wang; wanghp@cnu.edu.cn

Received 28 February 2014; Accepted 11 April 2014; Published 4 May 2014

Academic Editor: Józef Banaś

Copyright (C) $2014 \mathrm{H}$. Wang and Z. Wu. This is an open access article distributed under the Creative Commons Attribution License, which permits unrestricted use, distribution, and reproduction in any medium, provided the original work is properly cited.

Some sharp estimates of the $L_{p}(1 \leq p<\infty)$ modulus of continuity of classes of $\Lambda_{\varphi}$-bounded variation are obtained. As direct applications, we obtain estimates of order of Fourier coefficients of functions of $\Lambda_{\varphi}$-bounded variation, and we also characterize some sufficient and necessary conditions for the embedding relations $H_{p}^{\omega} \subset \Lambda_{\varphi} B V$. Our results include the corresponding known results of the class $\Lambda B V$ as a special case.

\section{Introduction and Main Results}

To generalize the notion of functions of bounded variation, Wiener [1] introduced the class $B V_{\beta}(\beta>1)$ of functions of $\beta$-bounded variation. Young [2] introduced the notion of functions of $\varphi$-bounded variation, and Waterman [3] studied a class of $\Lambda$-bounded variations. Combining the notion of $\Lambda$ bounded variation with that of $\varphi$-bounded variation, Leindler [4] introduced the class $\Lambda_{\varphi} B V$ of functions of $\Lambda_{\varphi}$-bounded variation, and both classes of $\Lambda$-bounded variation and $\varphi$ bounded variation are its special cases. Actually the class $\Lambda_{\varphi} B V$ first appeared in Schramm and Waterman's paper [5], and some restrictions are imposed on $\varphi$ in their definition. Here we adopt Leindler's definition.

Definition 1. Let $\varphi:[0, \infty) \rightarrow \mathbb{R}$ be a nondecreasing function with $\varphi(0)=0$, and let $\Lambda=:\left\{\lambda_{k}\right\}$ be a nondecreasing sequence of positive numbers such that $\sum_{k=1}^{\infty}\left(1 / \lambda_{k}\right)=$ $+\infty$. Let $\Gamma$ be the set of all sequences of nonoverlapping subintervals $\left[a_{k}, b_{k}\right]$ in $[a, b]$. If for any $\Delta=\left\{\left(a_{k}, b_{k}\right) \subset[a, b]\right.$, $\left.k=1,2, \ldots, n, n \in \mathbf{Z}^{+}\right\} \in \Gamma$, a real valued function $f$ : $[a, b] \rightarrow \mathbb{R}$ satisfies the condition

$$
\sum_{k=1}^{n} \frac{\varphi\left(\left|f\left(b_{k}\right)-f\left(a_{k}\right)\right|\right)}{\lambda_{k}}<\infty
$$

then $f$ is said to be of $\Lambda_{\varphi}$-bounded variation, and this fact is denoted by $f \in \Lambda_{\varphi} B V$. And the quantity

$$
V_{\Lambda_{\varphi}}(f ;[a, b]):=\sup _{\Delta \in \Gamma}\left\{\sum_{k=1}^{n} \frac{\varphi\left(\left|f\left(b_{k}\right)-f\left(a_{k}\right)\right|\right)}{\lambda_{k}}\right\}
$$

is said to be $\Lambda_{\varphi}$-total variation of $f$.

In the special case when $\varphi(x)=x^{\beta}(\beta \geq 1), f$ is said to be of $\Lambda_{\beta}$-bounded variation, and we write $f \in \Lambda_{\beta} B V$ and $V_{\Lambda_{\beta}}(f ;[a, b])=V_{\Lambda_{\varphi}}(f ;[a, b])$, and if $\beta=1, f$ is said to be of $\Lambda$-bounded variation, and we denote $f \in \Lambda B V$ and $V_{\Lambda}(f ;[a, b])=V_{\Lambda_{1}}(f ;[a, b])$.

In the case $\Lambda=\{1\}$, we get the class of $\varphi$-bounded variation, and $f$ is said to be of $\varphi$-bounded variation, and we denote $V_{\varphi}(f ;[a, b])=V_{\Lambda_{\varphi}}(f ;[a, b])$. More specifically, when $\varphi(x)=x^{\beta}(\beta \geq 1)$, we say that $f$ is of $\beta$-bounded variation, and we denote $f \in B V_{\beta}$ and $V_{\beta}(f ;[a, b])=V_{\varphi}(f ;[a, b])$. The class $B V_{\beta}$ is also called the Wiener class and $B V_{1}$ is the wellknown class of bounded variation $B V$.

It is easily seen from the definition that $\Lambda_{\varphi} B V$ functions are bounded; that is, $\Lambda_{\varphi} B V[a, b] \subseteq B[a, b]$, and the discontinuities of a $\Lambda_{\varphi} B V$ function are simple and therefore at most denumerable, where $B[a, b]$ denotes the class of bounded real valued functions on $[a, b]$.

Let $\Lambda=$ : $\left\{\lambda_{k}\right\}$ be a nondecreasing sequence of positive numbers such that $\sum_{k=1}^{\infty}\left(1 / \lambda_{k}\right)=+\infty$. If a continuous 
and nondecreasing function $\lambda(s)$ on $[0, \infty)$ such that $\lambda(s) \equiv$ $\lambda_{1}, 0 \leq s \leq 1$ and $\lambda(k)=\lambda_{k}, k=1,2, \ldots$, then we say that $\lambda(s)$ generates $\Lambda$. By the nondecreasing property of $\Lambda$, it is easily verified that if $\lambda(s)$ generates $\Lambda$, then

$$
\frac{1}{2} \int_{0}^{n} \frac{\mathrm{d} s}{\lambda(s)} \leq \sum_{k=1}^{n} \frac{1}{\lambda_{k}} \leq \int_{0}^{n} \frac{\mathrm{d} s}{\lambda(s)}, \quad n \geq 1 .
$$

Let $\lambda(x)$ be a nonnegative real-valued function on $[0, \infty)$. If there exists $a>0$ such that $\lambda(2 x) \leq a \lambda(x), x \in(0, A)$ for some positive constant $A$, then we say that $\lambda(x)$ satisfies the condition $\Delta_{2}$. If $\lambda(x)$ generates $\Lambda=\left\{\lambda_{k}\right\}$ and satisfies the condition $\Delta_{2}$, we say that $\Lambda$ satisfies the condition $\Delta_{2}$. Obviously the condition $\Delta_{2}$ here is a very weak restriction on $\lambda(x)$ and $\Lambda$.

Let $\omega(t)$ be a modulus of continuity, that is, a continuous and nondecreasing function on $[0,+\infty)$ satisfying $\omega(0)=0$ and $\omega\left(t_{1}+t_{2}\right) \leq \omega\left(t_{1}\right)+\omega\left(t_{2}\right)$ for nonnegative $t_{1}$ and $t_{2}$. As usual, for $1 \leq p \leq \infty$, denote by $H_{p}^{\omega} \equiv H_{p}^{\omega(t)}$ the class of functions for which $\|f\|_{H_{p}^{\omega}}:=\sup _{t>0}(\omega(f, t) p / \omega(t))<\infty$, where

$$
\begin{aligned}
& \omega(f ; t)_{p} \\
& := \begin{cases}\sup _{0 \leq h \leq t}\left\{\int_{a}^{b}|f(x+h)-f(x)|^{p} \mathrm{~d} x\right\}^{1 / p}, & 1 \leq p<\infty, \\
\sup _{0 \leq h \leq t} \sup _{x \in[a, b]}|f(x+h)-f(x)|, & p=\infty\end{cases}
\end{aligned}
$$

is the $L_{p}$ modulus of continuity of $f$. We write $H^{\omega}$ instead of $H_{\infty}^{\omega}$ and $H_{p}^{\alpha}(0<\alpha \leq 1)$ instead of $H_{p}^{t^{\alpha}} \equiv \operatorname{Lip}(\alpha, p)$, the Lipschitz class, for brevity.

Functions of classes $B V_{\beta}, \varphi B V, \Lambda B V$, and $\Lambda_{\varphi} B V$ are considered in trigonometric Fourier series and some of them share good approximative properties (see [1-3, 6-11], etc.). What we mention here is the following theorem proved by Shiba [12], Schramm and Waterman [5], and Wang [13]:

Theorem A. (a) If $f \in \Lambda_{\beta} B V, 1 \leq \beta<2 r, 1 \leq r<\infty$, and

$$
\frac{\sum_{n=1}^{\infty}\left(\sum_{k=1}^{n}\left(1 / \lambda_{k}\right)\right)^{-1 / 2 r}\left(\omega_{\beta+(2-\beta) s}(f ; \pi / n)\right)^{1-\beta / 2 r}}{n^{1 / 2}}<\infty,
$$

where $1 / r+1 / s=1$, then the Fourier series of $f$ converges absolutely.

(b) If $\varphi$ is $\Delta_{2}, f \in \Lambda_{\varphi} B V, 1 \leq \beta<2 r, 1 \leq r<\infty$, and

$$
\frac{\sum_{n=1}^{\infty}\left[\varphi^{-1}\left(\left(\sum_{k=1}^{n}\left(\frac{1}{\lambda_{k}}\right)\right)^{-1} \omega_{\beta+(2-\beta) s}^{2 r-\beta}\left(f ; \frac{\pi}{n}\right)\right)\right]^{1 / 2 r}}{n^{1 / 2}}<\infty,
$$

where $1 / r+1 / s=1$, then the Fourier series of $f$ converges absolutely.

Embedding relations between various generalized bounded variation classes and the class $H_{p}^{\omega}$ (or the Lipschitz class $H_{p}^{\alpha}$ ) are also investigated in recent years. It iswell known that $H_{p}^{1} \subset H_{1}^{1}=B V \subset \Lambda B V$. For $f \in \Lambda B V$, the estimates of $L_{p}$ modulus of continuity of $f$ had been given in [13] for $p=1$ and in $[9,14]$ for $1<p<\infty$. Furthermore, Goginava in [15] and Li and Wang in [9] proved that, for $1 \leq p<\infty$, $0<\alpha, \delta \leq 1$,

$$
\left\{n^{\delta}\right\} B V \subset H_{p}^{\alpha} \quad \text { iff } \alpha \leq \min \left\{\frac{1}{p}, 1-\delta\right\} .
$$

For more detailed results on this topic, we refer readers to [4, $9,10,14-25]$.

In this paper, we obtain some sharp estimates of $L_{p}(1 \leq$ $p<\infty)$ modulus of continuity of the classes $\Lambda_{\varphi} B V$ in the case of that $\varphi$ is convex. More specifically, our results include estimates of $L_{1}$ modulus of continuity of the classes $\Lambda_{\varphi} B V$, estimates of $L_{p}(1 \leq p<\infty)$ modulus of continuity of the classes $\Lambda_{\beta} B V$, and specially estimates of $L_{p}(1 \leq p<\infty)$ modulus of the classes $\left\{(n+1)^{\alpha} \ln ^{\gamma}(n+1)\right\}_{\beta} B V(0 \leq \alpha \leq 1, \beta \geq$ $1, \gamma \in \mathbb{R})$. Our results extend and include the corresponding known results of the class $\Lambda B V$ as a special case and are also sharp in most cases. As direct applications, we obtain estimates of order of Fourier coefficients of functions of $\Lambda_{\varphi}$ bounded variation, and we also characterize some sufficient and necessary conditions for the embedding relations $H_{p}^{\omega} \subset$ $\Lambda_{\varphi} B V$ and $H_{p}^{\omega} \subset \varphi B V$.

Now we state our main results, and in what follows, without loss of generality, we always assume that $[a, b]=$ $[0,2 \pi]$, and functions in various generalized bounded variation classes are $2 \pi$ periodic.

Theorem 2. Let $\Lambda_{\varphi} B V$ be the class offunctions of $\Lambda_{\varphi}$-bounded variation, and let $\lambda(s)$ generate $\Lambda$, where $\varphi$ is convex and $\varphi^{-1}$ is the inverse function of $\varphi$. Then, for $f \in \Lambda_{\varphi} B V$,

$$
\omega(f ; t)_{1} \leq 4 \pi \varphi^{-1}\left(\frac{V_{\Lambda_{\varphi}}(f ;[0,2 \pi])}{\int_{0}^{1 / t}(d s / \lambda(s))}\right),
$$

and this estimate is sharp in the sense of order provided that $\Lambda$ satisfies the condition $\Delta_{2}$.

Corollary 3 is a direct result of Theorem 2 by choosing $\varphi(x)=x$ and $\Lambda=\{1\}$, respectively.

Corollary 3. (a) Let $\Lambda B V$ be the class of functions of $\Lambda$ bounded variation, and let $\lambda(s)$ generate $\Lambda$. Then, for $f \in \Lambda B V$,

$$
\omega(f ; t)_{1} \leq \frac{4 \pi V_{\Lambda}(f ;[0,2 \pi])}{\int_{0}^{1 / t}(d s / \lambda(s))}
$$

and this estimate is sharp in the sense of order provided that $\Lambda$ satisfies the condition $\Delta_{2}$.

(b) Let $\varphi B V$ be the class of functions of $\varphi$-bounded variation, where $\varphi$ is convex. Then, for $f \in \varphi B V$,

$$
\omega(f ; t)_{1} \leq 4 \pi \varphi^{-1}\left(t V_{\varphi}(f ;[0,2 \pi])\right)
$$

and this estimate is sharp in the sense of order. 
The first part of Corollary 3(a) is due to Wang [13]. For special $\Lambda$ 's, one has

Corollary 4. Let $f \in \Lambda_{\varphi} B V$ and $\varphi$ be convex. Then

(a) for $\Lambda=\left\{n^{\alpha}\right\}(0 \leq \alpha<1)$,

$$
\omega(f ; t)_{1} \leq c \varphi^{-1}\left(t^{1-\alpha} V_{\Lambda_{\varphi}}(f ;[0,2 \pi])\right)
$$

(b) for $\Lambda=\{n\}$,

$$
\omega(f ; t)_{1} \leq c \varphi^{-1}\left(\frac{V_{\Lambda_{\varphi}}(f ;[0,2 \pi])}{\ln (1 / t)}\right) ;
$$

(c) for $\Lambda=\{(n+1) \ln (n+1)\}$,

$$
\omega(f ; t)_{1} \leq c \varphi^{-1}\left(\frac{V_{\Lambda_{\varphi}}(f ;[0,2 \pi])}{\ln \ln (1 / t)}\right)
$$

(d) for $\Lambda=\left\{\ln ^{\gamma}(n+1)\right\}(\gamma>0)$,

$$
\omega(f ; t)_{1} \leq c \varphi^{-1}\left(t \ln ^{-\gamma}\left(\frac{1}{t}\right) V_{\Lambda_{\varphi}}(f ;[0,2 \pi])\right) ;
$$

(e) for $\Lambda=\left\{(n+1) \ln ^{\gamma}(n+1)\right\}(-\infty<\gamma<1)$,

$$
\omega(f ; t)_{1} \leq c \varphi^{-1}\left(\frac{V_{\Lambda_{\varphi}}(f ;[0,2 \pi])}{\ln ^{1-\gamma}(1 / t)}\right) ;
$$

(f) for $\Lambda=\left\{(n+1)^{\alpha} \ln ^{\gamma}(n+1)\right\}(0<\alpha<1, \gamma \in \mathbb{R} \backslash\{0\})$,

$$
\omega(f ; t)_{1} \leq c \varphi^{-1}\left(t^{1-\alpha} \ln ^{-\gamma}\left(\frac{1}{t}\right) V_{\Lambda_{\varphi}}(f ;[0,2 \pi])\right) .
$$

The above estimates are sharp in the sense of order.

Let $f(x)$ be an integrable function on $[0,2 \pi]$ and let its Fourier coefficients be defined as follows:

$$
\begin{gathered}
a_{n}(f)=\frac{1}{\pi} \int_{0}^{2 \pi} f(t) \cos n t \mathrm{~d} t, \\
b_{n}(f)=\frac{1}{\pi} \int_{0}^{2 \pi} f(t) \sin n t \mathrm{~d} t, \\
c_{n}(f)=a_{n}(f)+i b_{n}(f) .
\end{gathered}
$$

We note that

$$
\begin{aligned}
\left|c_{n}(f)\right| & \leq \frac{1}{2 \pi} \int_{0}^{2 \pi}\left|f(t)-f\left(t+\frac{\pi}{n}\right)\right| \mathrm{d} t \\
& \leq c \omega_{1}\left(f ; \frac{1}{n}\right), \quad c>0 .
\end{aligned}
$$

Theorem 2 implies the following estimates of Fourier coefficients.
Corollary 5. (a) Let $\Lambda_{\varphi} B V$ be the class of functions of $\Lambda_{\varphi^{-}}$ bounded variation, and let $\lambda(s)$ generate $\Lambda$, where $\varphi$ is convex and $\varphi^{-1}$ is the inverse function of $\varphi$. Then, for $f \in \Lambda_{\varphi} B V$,

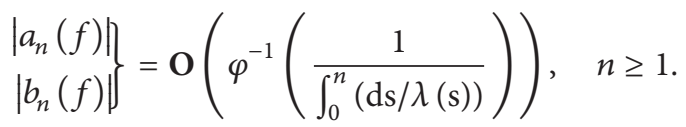

(b) Let $\varphi B V$ be the class of functions of $\varphi$-bounded variation, where $\varphi$ is convex and $\varphi^{-1}$ is the inverse function of $\varphi$. Then for $f \in \varphi B V$

$$
\left.\begin{array}{l}
\left|a_{n}(f)\right| \\
\left|b_{n}(f)\right|
\end{array}\right\}=\mathbf{O}\left(\varphi^{-1}\left(\frac{1}{n}\right)\right), \quad n \geq 1 .
$$

Corollary 5(a) includes Wang's result [13] for the class $\Lambda B V$ as a special case of $\varphi(x)=x$.

Theorem 6. Let $\Lambda_{\beta} B V(\beta \geq 1)$ be class of functions of $\Lambda_{\beta^{-}}$ bounded variation, and let $\lambda(s)$ generate $\Lambda=\left\{\lambda_{n}\right\}$. Set $\phi(z)=$ $\int_{0}^{z}(d s / \lambda(s))$ and assume $f \in \Lambda_{\beta} B V$.

(a) For $1 \leq p \leq \beta$,

$$
\omega(f ; t)_{p} \leq c\left(\frac{V_{\Lambda_{\beta}}(f ;[0,2 \pi])}{\int_{0}^{1 / t}(\mathrm{ds} / \lambda(\mathrm{s}))}\right)^{1 / \beta},
$$

and this estimate is sharp in the sense of order provided that $\Lambda$ satisfies the condition $\Delta_{2}$.

(b) For $1<p<\infty$,

$$
\omega(f ; t)_{p} \leq c\left(V_{\Lambda_{\beta}}(f ;[0,2 \pi])^{1 / \beta}\left(t \int_{0}^{1 / t} \frac{\mathrm{d} z}{\phi(z)^{p / \beta}}\right)^{1 / p} .\right.
$$

(c) If, for some $\beta<p<\infty, \phi(z)^{(\beta-p) / \beta} \lambda(z)$ is bounded on $[1, \infty)$, then

$$
\omega(f ; t)_{p} \leq c\left(V_{\Lambda_{\beta}}(f ;[0,2 \pi])^{1 / \beta} t^{1 / p} .\right.
$$

Unfortunately we cannot assert the sharpness of our estimates in (b) and (c) of Theorem 6 for the case $\beta<p<\infty$. Our next theorems concern some important special case of the class $\Lambda_{\beta} B V$ and elaborate on the estimates in Theorem 6. Among the classes considered here are $\left\{n^{\alpha}\right\}_{\beta} B V(0 \leq \alpha \leq$ $1, \beta \geq 1)$ and $\left\{(n+1)^{\alpha} \ln ^{\gamma}(n+1)\right\}_{\beta} B V(0 \leq \alpha \leq 1, \beta \geq 1, \gamma \in$ $\mathbb{R})$.

Theorem 7. (a) Let $\left\{n^{\alpha}\right\}_{\beta} B V(0 \leq \alpha \leq 1, \beta \geq 1)$ be the class of functions of $\left\{n^{\alpha}\right\}_{\beta}$-bounded variation. Then, for $f \in\left\{n^{\alpha}\right\}_{\beta} B V$ and $1 \leq p<\infty$, one has

(i) $\omega(f ; t)_{p} \leq c\left(V_{\Lambda_{\beta}}(f ;[0,2 \pi])\right)^{1 / \beta} t^{\min \{(1-\alpha) / \beta, 1 / p\}}, 0 \leq$ $\alpha<1$;

(ii) $\omega(f ; t)_{p} \leq c\left(V_{\Lambda_{\beta}}(f ;[0,2 \pi])\right)^{1 / \beta}\left(1 / \ln t^{-1}\right)^{1 / \beta}, \alpha=1$.

(b) Let $B V_{\beta}(\beta \geq 1)$ be the class of $\beta$-bounded variation, that is, the Wiener class. Then, for $f \in B V_{\beta}$ and $1 \leq p<\infty$,

$$
\omega(f ; t)_{p} \leq c\left(V_{\beta}(f ;[0,2 \pi])\right)^{1 / \beta} t^{\min \{1 / \beta, 1 / p\}},
$$

and both estimates in (a) and (b) are sharp in the sense of order. 
Li and Wang's results in [9] are extended in the Theorems 6 and 7 , which can be treated as the case $\beta=1$ of our theorems.

Theorem 8. Let $f \in \Lambda_{\beta} B V(\beta \geq 1)$. The following assertions are true.

(a) $\operatorname{For} \Lambda=\left\{\ln ^{\gamma}(n+1)\right\}(\gamma>0)$,

$$
\begin{aligned}
& \omega(f ; t)_{p} \\
& \quad \leq \begin{cases}c\left(V_{\Lambda_{\beta}}(f ;[0,2 \pi])\right)^{1 / \beta}\left(t \ln ^{\gamma}\left(\frac{1}{t}\right)\right)^{1 / \beta}, & 1 \leq p \leq \beta, \\
c\left(V_{\Lambda_{\beta}}(f ;[0,2 \pi])\right)^{1 / \beta} t^{1 / p}, & \beta<p<\infty .\end{cases}
\end{aligned}
$$

(b) For $\Lambda=\{(n+1) \ln (n+1)\}$,

$\omega(f ; t)_{p} \leq c\left(V_{\Lambda_{\beta}}(f ;[0,2 \pi])\right)^{1 / \beta}\left(\ln \ln \frac{1}{t}\right)^{-1 / \beta}, \quad 1 \leq p<\infty$.

(c) For $\Lambda=\left\{(n+1) \ln ^{\gamma}(n+1)\right\}(-\infty<\gamma<1)$,

$$
\begin{array}{r}
\omega(f ; t)_{p} \leq c\left(V_{\Lambda_{\beta}}(f ;[0,2 \pi])\right)^{1 / \beta}\left(\ln \frac{1}{t}\right)^{-(1-\gamma) / \beta}, \\
1 \leq p<\infty .
\end{array}
$$

(d) For $\Lambda=\left\{(n+1)^{\alpha} / \ln ^{\gamma}(n+1)\right\}(0<\alpha<1, \gamma>0)$,

$$
\begin{aligned}
& \omega(f ; t)_{p} \\
& \leq \begin{cases}c\left(V_{\Lambda_{\beta}}(f ;[0,2 \pi])\right)^{1 / \beta} t^{(1-\alpha) / \beta}\left(\ln \frac{1}{t}\right)^{-\gamma / \beta}, & 1 \leq p<\frac{\beta}{1-\alpha}, \\
c\left(V_{\Lambda_{\beta}}(f ;[0,2 \pi])\right)^{1 / \beta} t^{1 / p}, & p \geq \frac{\beta}{1-\alpha} .\end{cases}
\end{aligned}
$$

(e) For $\Lambda=\left\{(n+1)^{\alpha} \ln ^{\gamma}(n+1)\right\}(0<\alpha<1, \gamma>0)$

$$
\begin{aligned}
& \omega(f ; t)_{p} \\
& \leq \begin{cases}c\left(V_{\Lambda_{\beta}}(f ;[0,2 \pi])\right)^{1 / \beta} t^{(1-\alpha) / \beta}\left(\ln \frac{1}{t}\right)^{\gamma / \beta}, & 1 \leq p<\frac{\beta}{1-\alpha}, \\
c\left(V_{\Lambda_{\beta}}(f ;[0,2 \pi])\right)^{1 / \beta} t^{1 / p}, & p>\frac{\beta}{1-\alpha} .\end{cases}
\end{aligned}
$$

And all above estimates are sharp in the sense of order.

Remark 9. In (e), for $p=\beta /(1-\alpha)$, we only have

$$
\omega(f ; t)_{p} \leq c\left(V_{\Lambda_{\beta}}(f ;[0,2 \pi])\right)^{1 / \beta} t^{1 / p}\left(\ln \frac{1}{t}\right)^{1 / p+\gamma / \beta} .
$$

We do not know whether this estimate is sharp in the sense of order. However there exists $f_{n} \in \Lambda_{\beta} B V$ such that

$$
\begin{aligned}
& V_{\Lambda_{\beta}}\left(f_{n} ;[0,2 \pi]\right)=1, \quad n \longrightarrow+\infty \\
& \omega\left(f_{n} ; \frac{\pi}{n}\right)_{p} \geq c\left(\frac{\pi}{n}\right)^{1 / p}, \quad n \geq 10 .
\end{aligned}
$$

This exception indicates that the estimates of the $L_{p}$ modulus of continuity $\omega(f ; t)_{p}(\beta<p<+\infty)$ of classes of $\Lambda_{\beta^{-}}$ bounded variation are complicated, and Theorem 6 cannot cover all cases of the class $\Lambda_{\beta} B V(\beta \geq 1)$.

As direct applications of the above theorems, we characterize some sufficient and necessary conditions for the embedding relations between the generalized bounded variation classes and the class $H_{p}^{\omega}\left(\right.$ or $H_{p}^{\delta}$ ).

Corollary 10. On the embedding relations between the generalized bounded variation classes and the class $H_{p}^{\omega}$ or $H_{p}^{\delta}$, the following assertions are true.

(a) Let $\Lambda_{\varphi} B V$ be the class of functions of $\Lambda_{\varphi}$-bounded variation and let $\varphi$ be convex; let $\lambda(s)$ generate $\Lambda$ and $\Lambda$ satisfy the condition $\Delta_{2}$. Then

$$
\Lambda_{\varphi} B V \subset H_{1}^{\omega} \quad \text { iff } \omega(t)=\mathbf{O}\left(\varphi^{-1}\left(\left(\int_{0}^{1 / t} \frac{d s}{\lambda(s)}\right)^{-1}\right)\right)
$$$$
t \longrightarrow 0 \text {. }
$$

(b) Let $\varphi B V$ be the class of functions of $\varphi$-bounded variation and let $\varphi$ be convex. Then

$$
\varphi B V \subset H_{1}^{\omega} \quad \text { iff } \omega(t)=\mathbf{O}\left(\varphi^{-1}(t)\right), \quad t \longrightarrow 0
$$

(c) Let $\Lambda_{\beta} B V$ be the class of functions of $\Lambda_{\beta}$-bounded variation and let $\lambda(s)$ generate $\Lambda$.

(i) If, for $1 \leq p \leq \beta, \Lambda$ satisfies the condition $\Delta_{2}$, then

$$
\begin{array}{r}
\Lambda_{\beta} B V \subset H_{p}^{\omega} \quad \text { iff } \omega(t)=\mathbf{O}\left(\left(\int_{0}^{1 / t} \frac{d s}{\lambda(s)}\right)^{-1 / \beta}\right), \\
t \rightarrow 0 .
\end{array}
$$

(ii) If, for $\beta<p<\infty, \phi(z)^{(\beta-p) / \beta} \lambda(z)$ is bounded on $[1, \infty)$ and $0<\delta \leq 1 / p$, then $\Lambda_{\beta} B V \subset H_{p}^{\delta}$.

(d) Let $\left\{n^{\alpha}\right\}_{\beta} B V(0 \leq \alpha \leq 1, \beta \geq 1)$ be the class of functions of $\left\{n^{\alpha}\right\}_{\beta}$-bounded variation. Then, for $1 \leq$ $p<\infty$,

(i) $\left\{n^{\alpha}\right\}_{\beta} B V \subset H_{p}^{\delta}(0 \leq \alpha<1)$ if and only if $\delta \leq$ $\min \{(1-\alpha) / \beta, 1 / p\}$.

(ii) $\{n\}_{\beta} B V \subset H_{p}^{\omega}$ if and only if $\omega(t)=\mathbf{O}((\ln (1 /$ $\left.t))^{-1 / \beta}\right), t \rightarrow 0$.

(e) Let $B V_{\beta}(\beta \geq 1)$ be the class of functions of $\beta$-bounded variation, that is, Wiener class; then, for $1 \leq p<\infty$, $B V_{\beta} \subset H_{p}^{\delta}$ if and only if $\delta \leq \min \{1 / \beta, 1 / p\}$. 
This paper is organized as follows. In Section 2, we first state three lemmas, and then by them we prove Theorem 2 . Lemma 12 provides our proofs crucial upper estimates and Lemma 13 will be used repeatedly in proving the sharpness of our estimates. In Section 3, we prove Theorem 6, and the same estimate technique in [9] is partly used in our proof. Theorems 7 and 8 are proved in Section 4. For the case $\beta<$ $p<\infty$, the difficulty in our proofs of Theorems 7 and 8 is to prove the sharpness of our estimates and the key is to construct extreme functions by Lemma 13 .

\section{Proof of Theorem 2}

Before we start our proof of Theorem 2, we prove three lemmas. Lemmas 11 and 12 will also be used in the proof of Theorem 6. Lemma 13 will be employed repeatedly in the proof of the sharpness of our estimates. Lemmas 12 and 13 are of independent interest for functions of $\Lambda_{\varphi}$-bounded variation.

Lemma 11. Let $f \in L_{p}[a, b](p \geq 1)$. Then

$$
F(h)=\left(\int_{a}^{b}|f(x+h)-f(x)|^{p} d x\right)^{1 / p}
$$

is continuous on $[0, \infty)$.

Proof. Using triangle inequality of $L_{p}$ norm, for any $h_{1}, h_{2}>$ 0 , we have

$$
\begin{aligned}
\left|F\left(h_{1}\right)-F\left(h_{2}\right)\right| & \leq\left\|f\left(\cdot+h_{1}\right)-f\left(\cdot+h_{2}\right)\right\|_{p} \\
& \leq \omega\left(f ;\left|h_{1}-h_{2}\right|\right)_{p} .
\end{aligned}
$$

The right continuity of $\omega(f ; t)_{p}$ at $t=0$ implies the continuity of $F(h)$.

Lemma 12. Let $\Delta=\left\{\left[a_{k}, b_{k}\right]: 1 \leq k \leq n, n \in \mathbf{Z}^{+}\right\} \in \Gamma$ be an arbitrary sequence of nonoverlapping intervals in $[a, b]$, $\Lambda=\left\{\lambda_{k}\right\}, \varphi$ convex, and $\varphi^{-1}$ the function of $\varphi$. Then, for $f \in$ $\Lambda_{\varphi} B V$

$$
\sum_{k=1}^{n}\left|f\left(b_{k}\right)-f\left(a_{k}\right)\right| \leq n \varphi^{-1}\left(\frac{V_{\Lambda_{\varphi}}(f ;[a, b])}{\sum_{k=1}^{n} \lambda_{k}^{-1}}\right) .
$$

Specifically, if $f \in \varphi B V$, then $\sum_{k=1}^{n}\left|f\left(b_{k}\right)-f\left(a_{k}\right)\right| \leq$ $n \varphi^{-1}\left(V_{\varphi}(f ;[a, b]) / n\right)$.

Proof. By the definition of $\Lambda_{\varphi}$-total variation and letting $A_{i}=$ $1 / \lambda_{i}$ and $B_{k}=\varphi\left(\left|f\left(b_{k}\right)-f\left(a_{k}\right)\right|\right)$ in summation transform

$$
\begin{aligned}
\left(\sum_{i=1}^{n} A_{i}\right) & \left(\sum_{k=1}^{n} B_{k}\right) \\
= & \sum_{k=0}^{n-1}\left\{\sum_{i=1}^{n-k} A_{i} B_{i+k}+\sum_{i=n-k+1}^{n} A_{i} B_{i+k-n}\right\},
\end{aligned}
$$

we have

$$
\sum_{k=1}^{n} \varphi\left(\left|f\left(b_{k}\right)-f\left(a_{k}\right)\right|\right) \leq \frac{n}{\sum_{k=1}^{n} \lambda_{k}^{-1}} V_{\Lambda_{\varphi}}(f ;[a, b]) .
$$

Note that if $\varphi$ is an increasing convex function on $[0, \infty)$, then $\varphi^{-1}$ is increasing and concave on $[0, \infty)$. The concavity of $\varphi^{-1}$ implies that $\varphi^{-1}(a x) \geq a \varphi^{-1}(x), 0<a \leq 1$. Therefore, by Jensen's inequality and the above inequality, we finally get

$$
\begin{aligned}
\sum_{k=1}^{n}\left|f\left(b_{k}\right)-f\left(a_{k}\right)\right| \\
\quad=n \sum_{k=1}^{n} \frac{\varphi^{-1}\left(\varphi\left(\left|f\left(b_{k}\right)-f\left(a_{k}\right)\right|\right)\right)}{n} \\
\quad \leq n \varphi^{-1}\left(\frac{1}{n} \sum_{k=1}^{n} \varphi\left(\left|f\left(b_{k}\right)-f\left(a_{k}\right)\right|\right)\right) \\
\quad \leq n \varphi^{-1}\left(\frac{V_{\Lambda_{\varphi}}(f ;[a, b])}{\sum_{k=1}^{n} \lambda_{k}^{-1}}\right) .
\end{aligned}
$$

This completes the proof of Lemma 12.

Lemma 13. Let $\mathbf{a}_{n}=\left\{a_{k}\right\}_{k=1}^{n}$ be a given set of nonnegative and nonincreasing numbers. Define a function $f(x)$ on $[0,2 \pi]$ as

$$
f_{\mathbf{a}_{n}}(x)= \begin{cases}a_{i}, & \frac{(2 i-1) \pi}{n} \leq x<\frac{2 i \pi}{n}, \quad i=1,2, \ldots, n, \\ 0, & \text { other points of }[0,2 \pi],\end{cases}
$$

and extend it to $\mathbb{R}$ with period $2 \pi$. Then

(a) $f \in \Lambda_{\varphi} B V[0,2 \pi]$ and $V_{\Lambda_{\varphi}}\left(f_{\mathbf{a}_{n}} ;[0 ; 2 \pi]\right)=\sum_{i=1}^{n}$ $\left(1 / \lambda_{2 i-1}+1 / \lambda_{2 i}\right) \varphi\left(a_{i}\right)$

(b) $\omega\left(f_{\mathrm{a}_{n}} ; \pi / n\right)_{p}=\left((2 \pi / n) \sum_{k=1}^{n} a_{k}^{p}\right)^{1 / p}, p \geq 1$;

(c) $\sum_{i=1}^{n}\left(\varphi\left(a_{i}\right) / \lambda_{i}\right) \leq V_{\Lambda_{\varphi}}\left(f_{\mathbf{a}_{n}} ;[0 ; 2 \pi]\right) \leq 2 \sum_{i=1}^{n}\left(\varphi\left(a_{i}\right) / \lambda_{i}\right)$.

Proof. By the definition of $\Lambda_{\varphi}$-total variation and the nonnegative and nonincreasing properties of $\mathbf{a}_{n}$, direct computation proves (a). Since $\Lambda=\left\{\lambda_{k}\right\}$ is nondecreasing, it is obvious that (a) implies (c). For (b), computation shows that

$$
\begin{aligned}
\omega\left(f_{\mathbf{a}_{n}}\right. & \left.; \frac{\pi}{n}\right)_{p}^{p} \\
= & \sum_{k=1}^{n} \int_{(2(k-1) \pi) / n}^{2 k \pi / n}\left|f_{\mathbf{a}_{n}}\left(x+\frac{\pi}{n}\right)-f_{\mathbf{a}_{n}}(x)\right|^{p} \mathrm{~d} x \\
= & \frac{2 \pi}{n} \sum_{k=1}^{n} a_{k}^{p} .
\end{aligned}
$$

\section{Now we prove Theorem 2.}

Proof of Theorem 2. We write $V_{\Lambda_{\varphi}}(f)=V_{\Lambda_{\varphi}}(f ;[0,2 \pi])$ for simplicity. By Lemma 11, there exists an $h_{t} \in(0, t]$ such that

$$
\omega(f ; t)_{1}=\int_{0}^{2 \pi}\left|f\left(x+h_{t}\right)-f(x)\right| \mathrm{d} x .
$$


If we set $N=\left[2 \pi / h_{t}\right]$ and consider the periodicity of $f(x)$, then

$$
\omega(f ; t)_{1}=\frac{1}{N} \int_{0}^{2 \pi} F_{t}(x) \mathrm{d} x
$$

where

$$
F_{t}(x)=\sum_{k=1}^{N}\left|f\left(x+k h_{t}\right)-f\left(x+(k-1) h_{t}\right)\right| .
$$

From Jensen's inequality and Lemma 12 , for all $x \in[0,2 \pi]$, the concavity of $\varphi^{-1}$ implies

$$
F_{t}(x) \leq N \varphi^{-1}\left(\frac{V_{\Lambda_{\varphi}}(f)}{\sum_{k=1}^{N} \lambda_{k}^{-1}}\right) \leq 2 N \varphi^{-1}\left(\frac{V_{\Lambda_{\varphi}}(f)}{\int_{0}^{N}(\mathrm{~d} s / \lambda(s))}\right) .
$$

Substituting (46) into (44), we get

$$
\omega(f ; t)_{1} \leq 4 \pi \varphi^{-1}\left(\frac{V_{\Lambda_{\varphi}}(f)}{\int_{0}^{N}(\mathrm{~d} s / \lambda(s))}\right) .
$$

Since the right of (47) is decreasing with respect to $N$ and $N=\left[2 \pi / h_{t}\right] \geq[2 \pi / t] \geq 1 / t$, the estimate in Theorem 2 is obtained from (47) directly.

Now we show that our estimate is sharp in the sense of order under the assumption that $\Lambda$ satisfies the condition $\Delta_{2}$.

We choose

$$
a_{k}=\varphi^{-1}\left(\frac{1}{2 \pi \int_{0}^{n / \pi}(\mathrm{d} s / \lambda(s))}\right), \quad k=1, \ldots, n,
$$

and consider function $f_{\mathbf{a}_{n}}(x)$ defined in Lemma 13.

We have

$$
\begin{aligned}
\frac{\int_{0}^{n}(\mathrm{~d} s / \lambda(s))}{4 \int_{0}^{n}(\mathrm{~d} s / \lambda(s / \pi))} & =\frac{\int_{0}^{n}(\mathrm{~d} s / \lambda(s))}{4 \pi \int_{0}^{n / \pi}(\mathrm{d} s / \lambda(s))} \\
& \leq V_{\Lambda_{\varphi}}\left(f_{\mathrm{a}_{n}}\right) \\
& \leq \frac{\int_{0}^{n}(\mathrm{~d} s / \lambda(s))}{\pi \int_{0}^{n / \pi}(\mathrm{d} s / \lambda(s))} \\
& =\frac{\int_{0}^{n}(\mathrm{~d} s / \lambda(s))}{\int_{0}^{n}(\mathrm{~d} s / \lambda(s / \pi))}, \quad n \geq 10, \\
\omega\left(f_{\mathrm{a}_{n}} ; \frac{\pi}{n}\right)_{1}=2 \pi \varphi^{-1} & \left(\frac{1}{2 \pi \int_{0}^{n / \pi}(\mathrm{d} s / \lambda(s))}\right), \quad n \geq 10 .
\end{aligned}
$$

If $\Lambda$ satisfies the condition $\Delta_{2}$, that is, there exists $a>0$ such that $\lambda(2 x) \leq a \lambda(x), x>0$, then

$$
\lambda\left(\frac{s}{\pi}\right) \leq \lambda(s)=\lambda\left(\pi \cdot \frac{s}{\pi}\right) \leq \lambda\left(4 \cdot \frac{s}{\pi}\right) \leq a^{2} \lambda\left(\frac{s}{\pi}\right),
$$

and this yields

$$
\int_{0}^{n} \frac{\mathrm{d} s}{\lambda(s)} \leq \int_{0}^{n} \frac{\mathrm{d} s}{\lambda(s / \pi)} \leq a^{2} \int_{0}^{n} \frac{\mathrm{d} s}{\lambda(s)} .
$$

From (49) and (52), we have

$$
0<\frac{1}{4 a^{2}} \leq V_{\Lambda_{\varphi}}\left(f_{\mathbf{a}_{n}}\right) \leq 1, \quad n \geq 10 .
$$

On the other hand, (50), the concavity and the monotonicity of $\varphi^{-1}$ imply that

$$
\begin{aligned}
\omega\left(f_{\mathbf{a}_{n}} ; \frac{\pi}{n}\right)_{1} & \geq \varphi^{-1}\left(\frac{1}{\int_{0}^{n / \pi}(\mathrm{d} s / \lambda(s))}\right) \\
& \geq \varphi^{-1}\left(\frac{V_{\Lambda_{\varphi}}\left(f_{\mathbf{a}_{n}}\right)}{\int_{0}^{n / \pi}(\mathrm{d} s / \lambda(s))}\right), \quad n \geq 10 .
\end{aligned}
$$

Obviously, (53) and (54) mean the sharpness of our estimate in Theorem 2.

Proof of Corollary 4. Obviously the $\Lambda$ 's in Corollary 4 satisfy the condition $\Delta_{2}$. The proofs of (a), (b), (c), and (e) in Corollary 4 are obvious. In (d), we have

$$
\begin{gathered}
\lambda(s)=\ln ^{\gamma}(s+1), \quad s \geq 1, \\
\int_{0}^{z} \frac{\mathrm{d} s}{\lambda(s)} \asymp \frac{z}{\ln ^{\gamma} z}, \quad z \longrightarrow+\infty,
\end{gathered}
$$

and in (f), we have

$$
\begin{gathered}
\lambda(s)=(s+1)^{\alpha} \ln ^{\gamma}(s+1), \quad s \geq 1, \\
\int_{0}^{z} \frac{\mathrm{d} s}{\lambda(s)}=\frac{z^{1-\alpha}}{\ln ^{\gamma} z}, \quad z \longrightarrow+\infty,
\end{gathered}
$$

which complete the proofs of (d) and (f) of Corollary 4.

\section{Proof of Theorem 6}

In this section we prove Theorem 6. The proof of Theorem 6(a) is based on Hölder's inequality and Lemma 12. We use techniques used by Li and Wang [9] in the proofs of (b) and (c) of Theorem 6. Lemma 12 plays a crucial role in the whole proof of Theorem 6 .

Proof of Theorem 6. As in the proof of Theorem 2, it is easily seen from Lemma 11 that, for $1 \leq p<\infty$, there exists an $h_{t} \in(0, t]$ such that

$$
\omega(f ; t)_{p}^{p}=\frac{1}{N} \int_{0}^{2 \pi} F_{t}(x) \mathrm{d} x
$$

where

$$
\begin{array}{r}
F_{t}(x)=\sum_{k=1}^{N}\left|f\left(x+k h_{t}\right)-f\left(x+(k-1) h_{t}\right)\right|^{p}, \\
N=\left[\frac{2 \pi}{h_{t}}\right] .
\end{array}
$$


We first prove (a). We note that $r=\beta / p>1$ for $1 \leq p<\beta$. Let $s>0$ satisfy $1 / r+1 / s=1$. By Hölder's inequality and Lemma 12, we have

$$
\begin{aligned}
F_{t}(x) & \\
& \leq\left(\sum_{k=1}^{N}\left|f\left(x+k h_{t}\right)-f\left(x+(k-1) h_{t}\right)\right|^{\beta}\right)^{1 / r}\left(\sum_{k=1}^{N} 1^{s}\right)^{1 / s} \\
& \leq\left(\frac{N V_{\Lambda_{\beta}}(f)}{\sum_{k=1}^{N} \lambda_{k}^{-1}}\right)^{1 / r} N^{1 / s}=N\left(\frac{V_{\Lambda_{\beta}}(f)}{\sum_{k=1}^{N} \lambda_{k}^{-1}}\right)^{p / \beta} .
\end{aligned}
$$

For $p=\beta$, Lemma 12 directly yields

$$
F_{t}(x) \leq N\left(\frac{V_{\Lambda_{\beta}}(f)}{\sum_{k=1}^{N} \lambda_{k}^{-1}}\right) .
$$

Thus, for $1 \leq p \leq \beta$, we obtain

$$
F_{t}(x) \leq N\left(\frac{V_{\Lambda_{\beta}}(f)}{\sum_{k=1}^{N} \lambda_{k}^{-1}}\right)^{p / \beta} .
$$

Substituting (61) into (57) and noting that $N=\left[2 \pi / h_{t}\right] \geq$ $[2 \pi / t] \geq 1 / t$, we prove the desired estimate in Theorem $6(\mathrm{a})$.

If $\Lambda$ satisfies the condition $\Delta_{2}$, then from the proof of Theorem 2, we know that

$$
\int_{0}^{n} \frac{\mathrm{d} s}{\lambda(s)}=\int_{0}^{n / \pi} \frac{\mathrm{d} s}{\lambda(s)}, \quad n \longrightarrow \infty .
$$

If we choose

$$
\begin{aligned}
& a_{k}=\varepsilon_{n}^{1 / \beta}\left(\frac{1}{\int_{0}^{n / \pi}(\mathrm{d} s / \lambda(s))}\right)^{1 / \beta}, \quad k=1,2, \ldots, n, \\
& \text { where } \varepsilon_{n}=\frac{\int_{0}^{n / \pi}(\mathrm{d} s / \lambda(s))}{2 \int_{0}^{n}(\mathrm{~d} s / \lambda(s))}=1, \quad n \longrightarrow+\infty,
\end{aligned}
$$

and consider the functions $f_{\mathbf{a}_{n}}(x)$ defined in Lemma 13 , then we have

$$
\begin{aligned}
\frac{1}{4} & \leq V_{\Lambda_{\beta}}\left(f_{\mathbf{a}_{n}}\right) \leq 1, \quad n \geq 10, \\
\omega\left(f_{\mathbf{a}_{n}} ; \frac{\pi}{n}\right)_{p}^{p} & =2 \pi \varepsilon_{n}^{p / \beta}\left(\frac{1}{\int_{0}^{\pi / n}(\mathrm{~d} s / \lambda(s))}\right)^{p / \beta} \\
& \geq c\left(\frac{V_{\Lambda_{\beta}}\left(f_{\mathbf{a}_{n}}\right)}{\int_{0}^{\pi / n}(\mathrm{~d} s / \lambda(s))}\right)^{p / \beta}, \quad n \geq 10 .
\end{aligned}
$$

Equations (64) and (65) show that the estimate in Theorem 6(a) is sharp.
Now we prove (b). Without loss of generality, we assume that $\lambda_{1}=1$ and denote $\psi(x)=x^{\beta}(\beta \geq 1)$ and $\psi^{-1}(x)=x^{1 / \beta}$. From the definition of $V_{\Lambda_{\beta}}(f)$, we first have

$$
\left|f\left(x+k h_{t}\right)-f\left(x+(k-1) h_{t}\right)\right| \leq \psi^{-1}\left(V_{\Lambda_{\beta}}(f)\right) .
$$

Denote by $\sigma_{m}(m \geq 0)$ the set of integers $k(1 \leq k \leq N)$ for which

$$
\begin{aligned}
2^{-m-1} \psi^{-1}\left(V_{\Lambda_{\beta}}(f)\right) & <\left|f\left(x+k h_{t}\right)-f\left(x+(k-1) h_{t}\right)\right| \\
& \leq 2^{-m} \psi^{-1}\left(V_{\Lambda_{\beta}}(f)\right) .
\end{aligned}
$$

Then $\sum_{m=0}^{\infty}\left|\sigma_{m}\right|=N$, and there are at most $N$ nonempty $\sigma_{m}$, where $\left|\sigma_{m}\right|$ denotes the number of the elements in $\sigma_{m}$. Obviously $\left|\sigma_{m}\right| \leq N, m \geq 0$.

Hence we have

$$
\begin{aligned}
F_{t}(x) & =\sum_{m=0}^{\infty} \sum_{k \in \sigma_{m}}\left|f\left(x+k h_{t}\right)-f\left(x+(k-1) h_{t}\right)\right|^{p} \\
& \leq\left(\psi^{-1}\left(V_{\Lambda_{\beta}}(f)\right)\right)^{p} \sum_{m=0}^{\infty} 2^{-m p}\left|\sigma_{m}\right| \\
& =\left(V_{\Lambda_{\beta}}(f)\right)^{p / \beta}\left\{\sum_{m \leq M} 2^{-m p}\left|\sigma_{m}\right|+\sum_{m>M} 2^{-m p}\left|\sigma_{m}\right|\right\} \\
& =:\left(V_{\Lambda_{\beta}}(f)\right)^{p / \beta}\left(A_{1}+A_{2}\right),
\end{aligned}
$$

with $M>0$ to be determined.

From (67) and Lemma 12, we have, for $\left|\sigma_{m}\right| \neq 0$,

$$
\begin{aligned}
2^{-m-1} & \psi^{-1}\left(V_{\Lambda_{\beta}}(f)\right)\left|\sigma_{m}\right| \\
& \leq \sum_{k \in \sigma_{m}}\left|f\left(x+k h_{t}\right)-f\left(x+(k-1) h_{t}\right)\right| \\
& \leq\left|\sigma_{m}\right| \psi^{-1}\left(\frac{V_{\Lambda_{\beta}}(f)}{\sum_{i=1}^{\left|\sigma_{m}\right|} \lambda_{i}^{-1}}\right),
\end{aligned}
$$

and thus

$$
\sum_{i=1}^{\left|\sigma_{m}\right|} \frac{1}{\lambda_{i}} \leq \frac{V_{\Lambda_{\beta}}(f)}{\psi\left(2^{-m-1} \psi^{-1}\left(V_{\Lambda_{\beta}}(f)\right)\right)}=2^{\beta(m+1)} .
$$

Therefore,

$$
\phi\left(\left|\sigma_{m}\right|\right)=\int_{0}^{\left|\sigma_{m}\right|} \frac{\mathrm{d} s}{\lambda(s)} \leq 2 \sum_{i=1}^{\left|\sigma_{m}\right|} \frac{1}{\lambda_{i}} \leq 2^{\beta(m+1)+1} .
$$

If we set $z_{m}=\phi^{-1}\left(2^{\beta(m+1)+1}\right)$, where $\phi^{-1}$ is the inverse function of $\phi$, then

$$
\left|\sigma_{m}\right| \leq z_{m}, \quad 2^{-m}=\frac{2^{1+1 / \beta}}{\phi\left(z_{m}\right)^{1 / \beta}} .
$$


From the monotonicity of $\lambda(x)$, it is easily verified that $\phi(2 z) \leq 2 \phi(z)$. And from this we also have

$$
\phi\left(2 z_{m-1}\right) \leq 2 \phi\left(z_{m-1}\right)=2^{1-\beta} \phi\left(z_{m}\right) \leq \phi\left(z_{m}\right), \quad m \geq 1 .
$$

This yields

$$
2 z_{m-1} \leq z_{m}, \quad z_{m} \leq 2\left(z_{m}-z_{m-1}\right), \quad m \geq 1
$$

Now we estimate $A_{1}$ and $A_{2}$ in (68).

By means of (72) and (74), we first have

$$
\begin{aligned}
A_{1} & \leq \sum_{m \leq M} 2^{(2+1 / \beta) p} \frac{z_{m}}{\phi\left(z_{m}\right)^{p / \beta}} \\
& \leq 2^{(2+1 / \beta) p+1} \sum_{m \leq M} \frac{z_{m}-z_{m-1}}{\phi\left(z_{m}\right)^{p / \beta}} \\
& \leq c_{p} \int_{0}^{z_{M}} \frac{\mathrm{d} z}{\phi(z)^{p / \beta}} .
\end{aligned}
$$

If we choose $M=\left[\log _{2}^{\phi(N) / 2} / \beta\right]-1$, then $z_{M} \leq N \leq z_{M+1}$.

From (72) and the monotonicity of $\phi(z)$, we have

$$
\begin{aligned}
A_{2} & \leq N \sum_{m>M} 2^{-p m} \\
& =N \cdot \frac{2^{-p(M+1)}}{1-2^{-p}} \\
& \leq 2^{(1+1 / \beta) p+1} \frac{N}{\phi\left(z_{M+1}\right)^{p / \beta}} \\
& \leq c_{p} \frac{N}{\phi(N)^{p / \beta}} .
\end{aligned}
$$

Inserting (75) and (76) into (68) and noting that $\int_{0}^{N}(\mathrm{~d} z /$ $\left.\phi(z)^{p / \beta}\right) \geq\left(N / \phi(N)^{p / \beta}\right)$, for $x \in[0,2 \pi]$, we get

$$
\begin{aligned}
F_{t}(x) & \leq c_{3}\left(V_{\Lambda_{\beta}}(f)\right)^{p / \beta}\left[\int_{0}^{N} \frac{\mathrm{d} z}{\phi(z)^{p / \beta}}+\frac{N}{\phi(N)^{p / \beta}}\right] \\
& \leq 2 c_{p}\left(V_{\Lambda_{\beta}}(f)\right)^{p / \beta} \int_{0}^{N} \frac{\mathrm{d} z}{\phi(z)^{p / \beta}} .
\end{aligned}
$$

Combining (77) and (57), we finally obtain

$$
\omega(f ; t)_{p}^{p} \leq c_{p}\left(V_{\Lambda_{\beta}}(f)\right)^{p / \beta} \frac{1}{N} \int_{0}^{N} \frac{\mathrm{d} z}{\phi(z)^{p / \beta}} .
$$

Since $N=\left[2 \pi / h_{t}\right] \geq[2 \pi / t] \geq 1 / t$ and the right of $(78)$ is decreasing with respect to $N$, the proof of Theorem $6(\mathrm{~b})$ is complete.
Finally we prove Theorem 6(c). Notice that $\Lambda$ is nondecreasing. It follows from (67) that

$$
F_{t}(x)
$$

$$
\begin{aligned}
& =\sum_{m=0}^{\infty} \sum_{k \in \sigma_{m}}\left|f\left(x+k h_{t}\right)-f\left(x+(k-1) h_{t}\right)\right|^{p-\beta} \\
& \quad \times\left|f\left(x+k h_{t}\right)-f\left(x+(k-1) h_{t}\right)\right|^{\beta} \\
& \leq\left(V_{\Lambda_{\beta}}(f)\right)^{(p-\beta) / \beta} \\
& \quad \times \sum_{m=0}^{\infty} 2^{-m(p-\beta)} \sum_{k \in \sigma_{m}} \mid f\left(x+k h_{t}\right) \\
& \leq\left(V_{\Lambda_{\beta}}(f)\right)^{(p-\beta) / \beta} \sum_{m=0}^{\infty} 2^{-m(p-\beta)} \lambda_{k_{m}} \Omega_{m},
\end{aligned}
$$

where

$$
\begin{gathered}
k_{m}=\left|\sigma_{0}\right|+\left|\sigma_{1}\right|+\cdots+\left|\sigma_{m}\right| \\
\Omega_{m}=\sum_{j=k_{m-1}+1}^{k_{m}} \frac{\left|f\left(x+\sigma_{m}(j) h_{t}\right)-f\left(x+\left(\sigma_{m}(j)-1\right) h_{t}\right)\right|^{\beta}}{\lambda_{j}},
\end{gathered}
$$

and $\sigma_{m}(j) \in \sigma_{m}, k_{-1}=0$. It is obvious that $\sum_{m=0}^{\infty} \Omega_{m} \leq$ $V_{\Lambda_{\beta}}(f)$.

By (72) and (74), we know that

$$
k_{m} \leq \sum_{j=0}^{m} z_{j} \leq \sum_{j=1}^{m} 2\left(z_{j}-z_{j-1}\right)+z_{0} \leq 2 z_{m} .
$$

Assume that $\phi(z)^{(\beta-p) / \beta} \lambda(z)$ is bounded on $[1, \infty)$. From (72) and the fact $\phi\left(2 z_{m}\right) \leq 2 \phi\left(z_{m}\right), m \geq 0$, we obtain

$$
\begin{aligned}
2^{-m(p-\beta)} \lambda_{k_{m}} & \leq \frac{2^{(1+1 / \beta)(p-\beta)} \lambda\left(2 z_{m}\right)}{\phi\left(z_{m}\right)^{(p-\beta) / \beta}} \\
& \leq c \phi\left(2 z_{m}\right)^{(\beta-p) / \beta} \lambda\left(2 z_{m}\right) \leq c, \quad m \geq 0 .
\end{aligned}
$$

Finally, (79) yields

$$
\begin{aligned}
F_{t}(x) & \leq c\left(V_{\Lambda_{\beta}}(f)\right)^{(p-\beta) / \beta} \sum_{m=0}^{\infty} \Omega_{m} \\
& \leq c\left(V_{\Lambda_{\beta}}(f)\right)^{p / \beta}, \quad x \in[0,2 \pi] .
\end{aligned}
$$

Since $N \geq 1 / t$, we prove Theorem 6 (c) from (57). 


\section{Proofs of Theorems 7 and 8}

In this section, we prove Theorems 7 and 8.

Proof of Theorem 7. Theorem 7(a) implies Theorem 7(b) by letting $\alpha=0$. We only need to prove Theorem 7(a). Obviously $\Lambda=\left\{n^{\alpha}\right\}(0 \leq \alpha<1)$ satisfies the condition $\Delta_{2}$ and we have

$$
\begin{gathered}
\lambda(z)=z^{\alpha}, \quad z \geq 1 \\
\phi(z)=\int_{0}^{z} \frac{\mathrm{d} s}{\lambda(s)}=z^{1-\alpha}, \quad z \geq 1 .
\end{gathered}
$$

For $1 \leq p \leq \beta$, it follows from Theorem $6\left(\right.$ a) that $\omega(f ; t)_{p} \leq$ $c\left(V_{\Lambda_{\beta}}(f)\right)^{1 / \beta} t^{(1-\alpha) / \beta}$, and the order of this estimate is sharp.

For $\beta<p<\beta /(1-\alpha), 1-(p / \beta)(1-\alpha)>0$, we have

$$
\begin{array}{r}
\int_{0}^{N} \frac{\mathrm{d} z}{\phi(z)^{p / \beta}} \asymp \int_{1}^{N} \frac{\mathrm{d} z}{z^{p(1-\alpha) / \beta}}=N^{1-(p / \beta)(1-\alpha)}, \\
N \longrightarrow \infty .
\end{array}
$$

Substituting into Theorem $6(\mathrm{~b})$, we get $\omega(f ; t)_{p} \leq$ $c\left(V_{\Lambda_{\beta}}(f)\right)^{1 / \beta} t^{(1-\alpha) / \beta}$.

$$
\begin{aligned}
& \text { For } \beta /(1-\alpha) \leq p<\infty, \alpha+(1-\alpha)(\beta-p) / \beta \leq 0 \text {, we have } \\
& \phi(z)^{(\beta-p) / \beta} \lambda(z) \leq c_{1} z^{\alpha+(1-\alpha)(\beta-p) / \beta} \leq C<\infty, \quad z \geq 1 .
\end{aligned}
$$

By Theorem 6(c), we get the estimate

$$
\omega(f ; t)_{p} \leq\left(V_{\Lambda_{\beta}}(f)\right)^{1 / \beta} t^{1 / p} .
$$

If $\Lambda=\{n\}$, then $\Lambda$ satisfies the condition $\Delta_{2}$, and

$$
\begin{gathered}
\lambda(z)=z, \quad z \geq 1 \\
\phi(z)=\int_{0}^{z} \frac{\mathrm{d} s}{\lambda(s)} \asymp \ln (z+1) \asymp \ln z, \quad z \geq 1 .
\end{gathered}
$$

For $1 \leq p \leq \beta$, (ii) follows from Theorem 6(a) and the order in the estimate (ii) is also sharp.

For $\beta<p<\infty$, we have

$$
\begin{array}{r}
\frac{1}{N} \int_{0}^{N} \frac{\mathrm{d} z}{\phi(z)^{p / \beta}}=\frac{1}{N} \int_{1}^{N} \frac{\mathrm{d} z}{\ln (z+1)^{p / \beta}}=\frac{1}{(\ln z)^{p / \beta}}, \\
N \longrightarrow \infty .
\end{array}
$$

Substituting into Theorem 6(b), we get (ii).

Now we show the sharpness of the estimates in (i) and (ii) for $\beta<p<\infty$.

For this purpose, we consider $f_{\mathrm{a}_{n}}(x)$ defined in Lemma 13.

In (i), for $\beta<p<\beta /(1-\alpha)$, we choose

$$
a_{k}=\varepsilon_{n}^{1 / \beta}\left(\frac{\pi}{n}\right)^{(1-\alpha) / \beta}, \quad k=1,2, \ldots, n,
$$

where $\varepsilon_{n}=\frac{n^{1-\alpha}}{2 \pi^{1-\alpha} \sum_{k=1}^{n} k^{-\alpha}}=1, \quad n \longrightarrow \infty$.
From Lemma 13, we know that

$$
\begin{gathered}
\frac{1}{2} \leq V_{\Lambda_{\beta}}\left(f_{\mathbf{a}_{n}}\right) \leq 1, \quad n \geq 10, \\
\omega\left(f_{\mathbf{a}_{n}} ; \frac{\pi}{n}\right)_{p}^{p}=2 \pi \varepsilon_{n}^{p / \beta}\left(\frac{\pi}{n}\right)^{p(1-\alpha) / \beta} \\
\geq c\left(V_{\Lambda_{\beta}}\left(f_{\mathbf{a}_{n}}\right)\right)^{p / \beta}\left(\frac{\pi}{n}\right)^{p(1-\alpha) / \beta}, \quad n \geq 10 .
\end{gathered}
$$

For $\beta /(1-\alpha) \leq p<\infty, p(1-\alpha) / \beta \geq 1$, we choose

$$
\begin{array}{r}
a_{k}=\varepsilon_{n}^{1 / \beta} k^{-(1-\alpha) / \beta}(\ln (k+1))^{-(1+\sigma) / \beta}(\sigma>0), \\
k=1,2, \ldots, n,
\end{array}
$$

where

$$
\varepsilon_{n}=\left(2 \sum_{k=1}^{n} k^{-1}(\ln (k+1))^{-(1+\sigma)}\right)^{-1} \asymp 1, \quad n \longrightarrow+\infty
$$

From Lemma 13, we have

$$
\frac{1}{2} \leq V_{\Lambda_{\beta}}\left(f_{\mathbf{a}_{n}}\right) \leq 1, \quad n \geq 10,
$$

$$
\begin{aligned}
\omega\left(f_{\mathbf{a}_{n}} ; \frac{\pi}{n}\right)_{p}^{p} & =\frac{2 \pi}{n} \varepsilon_{n}^{p / \beta} \sum_{k=1}^{n} k^{-(1-\alpha) p / \beta}(\ln (1+k))^{-(1+\sigma) p / \beta} \\
& \geq c\left(V_{\Lambda_{\beta}}\left(f_{\mathbf{a}_{n}}\right)\right)^{p / \beta} \frac{\pi}{n}, \quad n \geq 10 .
\end{aligned}
$$

In (ii), for $\beta<p<\infty$, we choose

$$
a_{k}=\varepsilon_{n}^{1 / \beta}\left(\frac{n}{\pi}\right)^{1 / \beta}, \quad k=1,2, \ldots, n,
$$

where

$$
\varepsilon_{n}=\frac{\ln (n / \pi)}{2 \sum_{k=1}^{n} k^{-1}} \asymp 1, \quad n \longrightarrow+\infty
$$

From Lemma 13, we know that

$$
\begin{gathered}
\frac{1}{2} \leq V_{\Lambda_{\beta}}\left(f_{\mathbf{a}_{n}}\right) \leq 1, \quad n \geq 10, \\
\omega\left(f_{\mathbf{a}_{n}} ; \frac{\pi}{n}\right)_{p}^{p}=2 \pi \varepsilon_{n}^{p / \beta}\left(\ln \left(\frac{n}{\pi}\right)\right)^{-p / \beta} \\
\geq c\left(V_{\Lambda_{\beta}}\left(f_{\mathrm{a}_{n}}\right)\right)^{-p / \beta}\left(\ln \frac{n}{\pi}\right)^{-p / \beta}, \quad n \geq 10 .
\end{gathered}
$$

Obviously the above functions $f_{\mathrm{a}_{n}}$ chosen prove the sharpness of our estimates in Theorem 7 for $\beta<p<\infty$.

The proof of Theorem 8 is similar to that of Theorem 7 , but computations are more complicated. 
Proof of Theorem 8. Let $\lambda(s)$ generate $\Lambda$ and $\phi(z)=\int_{0}^{z}(\mathrm{~d} s /$ $\lambda(s))$. It is readily seen that $\Lambda$ 's in (a), (b), (c), (d), and (e) satisfy the condition $\Delta_{2}$.

In (a), we have

$$
\begin{aligned}
& \lambda(s)=\ln ^{\gamma}(s+1) \quad(\gamma>0), \quad s \geq 1, \\
& \phi(z)=\frac{z+1}{\ln ^{\gamma}(z+1)}=\frac{z}{\ln ^{\gamma} z}, \quad z \geq 2 .
\end{aligned}
$$

In (b), we have

$$
\begin{gathered}
\lambda(s)=(s+1) \ln (s+1), \quad s \geq 1, \\
\phi(z)=\ln \ln (z+1)=\ln \ln z, \quad z \geq 10 .
\end{gathered}
$$

In (c), we have

$$
\begin{gathered}
\lambda(s)=(s+1) \ln ^{\gamma}(s+1) \quad(-\infty<\gamma<1), \quad s \geq 1, \\
\phi(z)=(\ln (z+1))^{1-\gamma}=(\ln z)^{1-\gamma}, \quad z \geq 2 .
\end{gathered}
$$

In (d), we have

$$
\begin{gathered}
\lambda(s)=\frac{(s+1)^{\alpha}}{\ln ^{\gamma}(s+1)} \quad(0<\alpha<1, \gamma>0), s \geq 1, \\
\phi(z)=(z+1)^{1-\alpha}\left(\ln ^{\gamma}(z+1)\right)=z^{1-\alpha} \ln ^{\gamma} z, \quad z \geq 2 .
\end{gathered}
$$

In (e), we have

$$
\begin{gathered}
\lambda(s)=(s+1)^{\alpha} \ln ^{\gamma}(s+1) \quad(0<\alpha<1, \gamma>0), \quad s \geq 2, \\
\phi(z)=\frac{(z+1)^{1-\alpha}}{\ln ^{\gamma}(z+1)}=\frac{z^{1-\alpha}}{\ln ^{\gamma} z}, \quad z \geq 2 .
\end{gathered}
$$

From Theorem 6(a) we obtain sharp estimates in (a), (b), (c), (d), and (e) for $1 \leq p \leq \beta$.

Now we prove the estimates in Theorem 8 for $\beta<p<$ $\infty$. We apply (b) and (c) in Theorem 6 for upper estimates. The technique used here to show the sharpness is the same as that of the proof of Theorem 7 and the key is to choose $\mathbf{a}_{n}$ to construct extreme functions $f_{\mathbf{a}_{n}}(x)$ defined in Lemma 13.

In (a), for $\beta<p<\infty$, we have

$$
\phi(z)^{(\beta-p) / \beta} \lambda(z)=\frac{(\ln (z+1))^{p \gamma / \beta}}{(z+1)^{(p-\beta) / \beta}}<+\infty, \quad z \geq 1 .
$$

From Theorem 6(b), we obtain

$$
\omega(f ; t)_{p} \leq c\left(V_{\Lambda_{\beta}}(f)\right)^{1 / \beta} t^{1 / p} .
$$

If we choose

$$
\begin{array}{r}
a_{k}=\varepsilon_{n}^{1 / \beta}(k+1)^{-1 / \beta}(\ln (k+1))^{-(1+\gamma+\sigma) / \beta} \\
(\sigma>0), \quad k=1,2, \ldots, n,
\end{array}
$$

where

$$
\varepsilon_{n}=\left(2 \sum_{k=1}^{n}(k+1)^{-1}(\ln (k+1))^{-(1+\sigma)}\right)^{-1}=1, \quad n \longrightarrow+\infty,
$$

then

$$
\begin{gathered}
\frac{1}{2} \leq V_{\Lambda_{\beta}}\left(f_{\mathrm{a}_{n}}\right) \leq 1, \quad n \geq 10 \\
\omega\left(f_{\mathrm{a}_{n}} ; \frac{\pi}{n}\right)_{p}^{p}=2 \varepsilon_{n}^{p / \beta} \sum_{k=1}^{n}\left((k+1)^{-p / \beta}(\ln (k+1))^{-(p(1+\sigma) / \beta)}\right) \\
\cdot \frac{\pi}{n} \geq c\left(V_{\Lambda_{\beta}}\left(f_{\mathrm{a}_{n}}\right)\right)^{p / \beta} \cdot \frac{\pi}{n}, \quad n \geq 10 .
\end{gathered}
$$

Equations (107) and (108) imply the sharpness of (104).

In (b), we have

$$
\begin{aligned}
\frac{1}{N} \int_{0}^{N} \frac{\mathrm{d} z}{\phi(z)^{p / \beta}} & =\frac{1}{N} \int_{1}^{N} \frac{\mathrm{d} z}{(\ln \ln (z+1))^{p / \beta}} \\
& =\frac{1}{(\ln \ln N)^{p / \beta}}, \quad N \longrightarrow+\infty .
\end{aligned}
$$

Again from Theorem 6(b), we have

$$
\omega(f ; t)_{p} \leq c\left(V_{\Lambda_{\beta}}(f)\right)^{1 / \beta}\left(\ln \ln \frac{1}{t}\right)^{-1 / \beta} .
$$

If we choose

$$
a_{k}=\varepsilon_{n}^{1 / \beta}\left(\ln \ln \frac{n}{\pi}\right)^{-1 / \beta}, \quad k=1,2, \ldots, n,
$$

where

$$
\varepsilon_{n}=\frac{\ln \ln (n / \pi)}{2 \sum_{k=1}^{n}[(k+1)(\ln (k+1))]^{-1}}=1, \quad n \longrightarrow+\infty,
$$

then

$$
\begin{aligned}
\frac{1}{2} \leq V_{\Lambda_{\beta}}\left(f_{\mathbf{a}_{n}}\right) \leq 1, \quad n \geq 10, \\
\omega\left(f_{\mathbf{a}_{n}} ; \frac{\pi}{n}\right)_{p}^{p}=\frac{2 \pi}{n} \varepsilon_{n}^{p / \beta} \sum_{k=1}^{n}\left(\ln \ln \frac{n}{\pi}\right)^{-p / \beta} \\
\geq c\left(V_{\Lambda_{\beta}}\left(f_{\mathbf{a}_{n}}\right)\right)^{p / \beta}\left(\ln \ln \frac{n}{\pi}\right)^{-p / \beta}, \quad n \geq 10 .
\end{aligned}
$$

Equations (113) and (114) imply the sharpness of (110). In (c), for $\beta<p<\infty$, we have

$$
\begin{aligned}
\frac{1}{N} \int_{0}^{N} \frac{\mathrm{d} z}{\phi(z)^{p / \beta}} & =\frac{1}{N} \int_{1}^{N} \frac{\mathrm{d} z}{(\ln (z+1))^{p(1-\gamma) / \beta}} \\
& =\frac{1}{(\ln N)^{p(1-\gamma) / \beta}}, \quad N \rightarrow \infty .
\end{aligned}
$$

From Theorem 6(b), we get

$$
\omega(f ; t)_{p} \leq c\left(V_{\Lambda_{\beta}}(f)\right)^{1 / \beta}\left(\ln \frac{1}{t}\right)^{-(1-\gamma) / \beta} .
$$


If we choose

$$
a_{k}=\varepsilon_{n}^{1 / \beta}\left(\ln \frac{n}{\pi}\right)^{-(1-\gamma) / \beta}, \quad k=1,2, \ldots, n,
$$

where

$$
\varepsilon_{n}=\frac{(\ln (n / \pi))^{1-\gamma}}{2 \sum_{k=1}^{n}(k+1)^{-1}(\ln (k+1))^{-\gamma}}=1, \quad n \longrightarrow+\infty,
$$

then

$$
\begin{gathered}
\frac{1}{2} \leq V_{\Lambda_{\beta}}\left(f_{\mathrm{a}_{n}}\right) \leq 1, \quad n \geq 10 \\
\omega\left(f_{\mathrm{a}_{n}} ; \frac{\pi}{n}\right)_{p}^{p}=2 \pi \varepsilon_{n}^{p / \beta}\left(\ln \frac{n}{\pi}\right)^{-p(1-\gamma) / \beta} \\
\geq c\left(V_{\Lambda_{\beta}}\left(f_{\mathrm{a}_{n}}\right)\right)^{p / \beta}\left(\ln \frac{n}{\pi}\right)^{-p(1-\gamma) / \beta}, \quad n \geq 10 .
\end{gathered}
$$

Equations (119) and (120) imply the sharpness of (116).

In (d), for $\beta<p<\beta /(1-\alpha)$, we have

$$
\begin{aligned}
\frac{1}{N} \int_{0}^{N} \frac{\mathrm{d} z}{\phi(z)^{p / \beta}} & =\frac{1}{N} \int_{1}^{N} \frac{\mathrm{d} z}{(z+1)^{p(1-\alpha) / \beta}(\ln (z+1))^{p \gamma / \beta}} \\
& =\frac{1}{N^{p(1-\alpha) / \beta}(\ln N)^{p \gamma / \beta}}, \quad N \longrightarrow \infty .
\end{aligned}
$$

From Theorem 6(b), we get

$$
\omega(f ; t)_{p} \leq c\left(V_{\Lambda_{\beta}}(f)\right)^{1 / \beta} t^{(1-\alpha) / \beta}\left(\ln \frac{1}{t}\right)^{-\gamma / \beta} .
$$

If we choose

$$
a_{k}=\varepsilon_{n}^{1 / \beta}\left(\frac{\pi}{n}\right)^{(1-\alpha) / \beta}\left(\ln \frac{n}{\pi}\right)^{-\gamma / \beta}, \quad k=1,2, \ldots, n,
$$

where

$$
\varepsilon_{n}=\frac{(n / \pi)^{1-\alpha}(\ln (n / \pi))^{\gamma}}{2 \sum_{k=1}^{n}(k+1)^{-\alpha} \ln ^{\gamma}(k+1)}=1, \quad n \longrightarrow+\infty,
$$

then

$$
\begin{gathered}
\frac{1}{2} \leq V_{\Lambda_{\beta}}\left(f_{\mathrm{a}_{n}}\right) \leq 1, \quad n \geq 10, \\
\omega\left(f_{\mathrm{a}_{n}} ; \frac{\pi}{n}\right)_{p}^{p}=2 \pi \varepsilon_{n}^{p / \beta}\left(\frac{\pi}{n}\right)^{p(1-\gamma) / \beta}\left(\ln \frac{n}{\pi}\right)^{-p \gamma / \beta} \\
\geq c\left(V_{\Lambda_{\beta}}\left(f_{\mathrm{a}_{n}}\right)\right)^{p / \beta}\left(\frac{\pi}{n}\right)^{p(1-\gamma) / \beta}\left(\ln \frac{n}{\pi}\right)^{-p \gamma / \beta}, \\
n \geq 10 .
\end{gathered}
$$

Equations (125) and (126) imply the sharpness of (122).
For $\beta /(1-\alpha) \leq p<\infty, \beta-p(1-\alpha) \leq 0$, we have

$$
\phi(z)^{(\beta-p) / \beta} \lambda(z)=\frac{(z+1)^{\beta-p(1-\alpha) / \beta}}{(\ln (z+1))^{p \gamma / \beta}}<+\infty, \quad z \geq 1 .
$$

From Theorem 6(c), we get

$$
\omega(f ; t)_{p} \leq c\left(V_{\Lambda_{\beta}}(f)\right)^{1 / \beta} t^{1 / p} .
$$

If we choose

$$
a_{k}=\varepsilon_{n}^{1 / \beta}(k+1)^{-(1-\alpha+\sigma) / \beta}(\ln (k+1))^{-\gamma / \beta}
$$

$$
(\sigma>0), \quad k=1,2, \ldots, n,
$$

where

$$
\varepsilon_{n}=\left(2 \sum_{k=1}^{n}(k+1)^{-(1+\sigma)}\right)^{-1}=1, \quad n \longrightarrow+\infty,
$$

then

$$
\begin{gathered}
\frac{1}{2} \leq V_{\Lambda_{\beta}}\left(f_{\mathrm{a}_{n}}\right) \leq 1, \quad n \geq 10, \\
\omega\left(f_{\mathbf{a}_{n}} ; \frac{\pi}{n}\right)_{p}^{p}=2 \varepsilon_{n}^{p / \beta} \sum_{k=1}^{n}\left((k+1)^{-(p(1-\alpha+\sigma) / \beta)}(\ln (k+1))^{-p \gamma / \beta}\right) \\
\cdot \frac{\pi}{n} \geq c\left(V_{\Lambda_{\beta}}\left(f_{\mathrm{a}_{n}}\right)\right)^{p / \beta} \cdot \frac{\pi}{n}, \quad n \geq 10 .
\end{gathered}
$$

Equations (131) and (132) imply the sharpness of (128).

In (e), for $\beta<p<\beta /(1-\alpha)$, we have

$$
\begin{aligned}
\frac{1}{N} \int_{0}^{N} \frac{\mathrm{d} z}{\phi(z)^{p / \beta}} & =\frac{1}{N} \int_{1}^{N} \frac{(\ln (z+1))^{p \gamma / \beta}}{(z+1)^{p(1-\alpha) / \beta}} \mathrm{d} z \\
& =\frac{(\ln N)^{p \gamma / \beta}}{N^{p^{(1-\alpha) / \beta}}}, \quad N \longrightarrow \infty .
\end{aligned}
$$

From Theorem 6(b), we get

$$
\omega(f ; t)_{p} \leq c\left(V_{\Lambda_{\beta}}(f)\right)^{1 / \beta} t^{(1-\alpha) / \beta}\left(\ln \frac{1}{t}\right)^{\gamma / \beta} .
$$

If we choose

$$
a_{k}=\varepsilon_{n}^{1 / \beta}\left(\frac{\pi}{n}\right)^{(1-\alpha) / \beta}\left(\ln \frac{n}{\pi}\right)^{\gamma / \beta}, \quad k=1,2, \ldots, n,
$$

where

$$
\varepsilon_{n}=\frac{(n / \pi)^{1-\alpha}(\ln (n / \pi))^{-\gamma}}{2 \sum_{k=1}^{n}(k+1)^{-\alpha}(\ln (k+1))^{-\gamma}}=1 \quad n \longrightarrow+\infty,
$$

then

$$
\begin{gathered}
\frac{1}{2} \leq V_{\Lambda_{\beta}}\left(f_{\mathbf{a}_{n}}\right) \leq 1, \quad n \geq 10, \\
\omega\left(f_{\mathbf{a}_{n}} ; \frac{\pi}{n}\right)_{p}^{p}=2 \pi \varepsilon_{n}^{p / \beta}\left(\frac{n}{\pi}\right)^{p(1-\gamma) / \beta}\left(\ln \frac{n}{\pi}\right)^{p \gamma / \beta} \\
\geq c\left(V_{\Lambda_{\beta}}\left(f_{\mathbf{a}_{n}}\right)\right)^{p / \beta}\left(\frac{n}{\pi}\right)^{p(1-\gamma) / \beta}\left(\ln \frac{n}{\pi}\right)^{p \gamma / \beta}, \\
n \geq 10 .
\end{gathered}
$$

Equations (137) and (138) imply the sharpness of (134). 
For $\beta /(1-\alpha)<p<\infty, \beta-p(1-\alpha)<0$, we have

$$
\phi(z)^{(\beta-p) / \beta} \lambda(z)=\frac{(\ln (z+1))^{p \gamma / \beta}}{(z+1)^{(p(1-\alpha)-\beta) / \beta}}<+\infty, \quad z \geq 1 .
$$

From Theorem 6(c), we get

$$
\omega(f ; t)_{p} \leq c\left(V_{\Lambda_{\beta}}(f)\right)^{1 / \beta} t^{1 / p} .
$$

If we choose

$$
\begin{array}{r}
a_{k}=\varepsilon_{n}^{1 / \beta}(k+1)^{-(1-\alpha+\sigma) / \beta}(\ln (k+1))^{\gamma / \beta} \\
(\sigma>0), \quad k=1,2, \ldots, n,
\end{array}
$$

where

$$
\varepsilon_{n}=\left(2 \sum_{k=1}^{n}(k+1)^{-(1+\sigma)}\right)^{-1} \asymp 1, \quad n \longrightarrow+\infty,
$$

then

$$
\begin{gathered}
\frac{1}{2} \leq V_{\Lambda_{\beta}}\left(f_{\mathrm{a}_{n}}\right) \leq 1, \quad n \geq 10 \\
\omega\left(f_{\mathbf{a}_{n}} ; \frac{\pi}{n}\right)_{p}^{p}=2 \varepsilon_{n}^{p / \beta} \sum_{k=1}^{n}(k+1)^{-p(1-\alpha+\sigma) / \beta}(\ln (k+1))^{p \gamma / \beta} \\
\cdot \frac{\pi}{n} \geq c\left(V_{\Lambda_{\beta}}\left(f_{\mathbf{a}_{n}}\right)\right)^{p / \beta} \cdot \frac{\pi}{n}, \quad n \geq 10 .
\end{gathered}
$$

Equations (143) and (144) imply the sharpness of (140).

But, for $p=\beta /(1-\alpha)$, we have

$$
\begin{aligned}
& \phi(z)^{(\beta-p) / \beta} \lambda(z)=(\ln (z+1))^{p \gamma / \beta} \longrightarrow+\infty, \\
& \text { as } z \longrightarrow+\infty, \\
& \frac{1}{N} \int_{0}^{N} \frac{\mathrm{d} z}{\phi(z)^{p / \beta}}=\frac{1}{N} \int_{1}^{N} \frac{(\ln (z+1))^{p \gamma / \beta}}{z+1} \mathrm{~d} z=\frac{(\ln N)^{1+p \gamma / \beta}}{N}, \\
& N \longrightarrow+\infty .
\end{aligned}
$$

Theorem 6(c) is not applicable for this case. From Theorem 6(b), we obtain

$$
\omega(f ; t)_{p} \leq c\left(V_{\Lambda_{\beta}}(f ;[0,2 \pi])\right)^{1 / \beta} t^{1 / p}\left(\ln \frac{1}{t}\right)^{1 / p+\gamma / \beta} .
$$

Unfortunately this estimate is not sharp in the sense of order. However, if we choose

$$
\begin{aligned}
a_{k}= & \varepsilon_{n}^{1 / \beta}(k+1)^{-(1-\alpha) / \beta}(\ln (k+1))^{-(1-\gamma+\sigma) / \beta} \\
& (\sigma>\max \{0, \gamma-\alpha\}), \quad k=1,2, \ldots, n,
\end{aligned}
$$

where

$$
\varepsilon_{n}=\left[\sum_{k=1}^{n}(k+1)^{-1}(\ln (k+1))^{-(1+\sigma)}\right]^{-1} \asymp 1, \quad n \longrightarrow+\infty,
$$

then we have

$$
\begin{gathered}
\frac{1}{2} \leq V_{\Lambda_{\beta}}\left(f_{\mathbf{a}_{n}}\right) \leq 1, \quad n \geq 10, \\
\omega\left(f_{\mathbf{a}_{n}} ; \frac{\pi}{n}\right)_{p}^{p}=2 \varepsilon_{n}^{p / \beta} \sum_{k=1}^{n}(k+1)^{-1}(\ln (k+1))^{-(1-\gamma+\sigma) /(1-\alpha)} \\
\cdot \frac{\pi}{n} \geq c \cdot \frac{\pi}{n}, \quad n \geq 10 .
\end{gathered}
$$

In other words, there exists $f_{n} \in \Lambda_{\beta} B V$ such that

$$
\begin{gathered}
V_{\Lambda_{\beta}}(f)=1, \quad n \longrightarrow+\infty, \\
\omega\left(f_{n} ; \frac{\pi}{n}\right)_{p} \geq c\left(\frac{\pi}{n}\right)^{1 / p}, \quad n \geq 10 .
\end{gathered}
$$

This exception indicates that our methods used in this paper cannot cover all cases of estimates of $L_{p}(1 \leq p<\infty)$ modulus of continuity of classes of functions of $\Lambda_{\beta}$-bounded variation.

Proof of Corollary 10. Obviously Theorem 2 implies (a) and (b). (c) follows from Theorem 6. Finally, (d) and (e) are obtained from Theorem 7 directly.

\section{Conflict of Interests}

The authors declare that there is no conflict of interests regarding the publication of this paper.

\section{Acknowledgments}

The authors would like to thank Professor Zhongkai Li for various helpful discussions. Heping Wang is supported by the National Natural Science Foundation of China (Project no. 11271263), the Beijing Natural Science Foundation (1132001), and BCMIIS.

\section{References}

[1] N. Wiener, "The quadratic variation of a function and its Fourier coefficients," Massachusetts Journal of Mathematics, vol. 3, pp. 72-94, 1924.

[2] L. C. Young, "Sur une généralisation de la notion de variation de puissance pième bornée au sens de $\mathrm{M}$. Wiener, et sur la convergence des séries de Fourier," Comptes Rendus de Lacadémie des Sciences, vol. 204, pp. 470-472, 1937.

[3] D. Waterman, "On convergence of Fourier series of functions of generalized bounded variation," Studia Mathematica, vol. 44, pp. 107-117, 1972.

[4] L. Leindler, "A note on embedding of classes $H^{\omega}$," Analysis Mathematica, vol. 27, no. 1, pp. 71-76, 2001.

[5] M. Schramm and D. Waterman, "Absolute convergence of Fourier series of functions of $\Lambda B V^{(p)}$ and $\phi \Lambda B V$," Acta Mathematica Academiae Scientiarum Hungaricae, vol. 40, no. 3-4, pp. 273$276,1982$.

[6] J. Musielak and W. Orlicz, "On generalized variations. I," Studia Mathematica, vol. 18, pp. 11-41, 1959. 
[7] D. Waterman, "On the summability of Fourier series of functions of L-bounded variation," Studia Mathematica, vol. 54, no. 1, pp. 87-95, 1976.

[8] S. Perlman and D. Waterman, "Some remarks on functions of $\Lambda$ bounded variation," Proceedings of the American Mathematical Society, vol. 74, no. 1, pp. 113-123, 1979.

[9] Z. Li and H. Wang, "Estimates of $L^{p}$-continuity modulus of $\Lambda \mathrm{BV}$ functions and applications in Fourier series," Applicable Analysis, vol. 90, no. 3-4, pp. 475-482, 2011.

[10] H. Wang, "Embedding of Lipschitz classes into classes of functions of $\Lambda$-bounded variation," Journal of Mathematical Analysis and Applications, vol. 354, no. 2, pp. 698-703, 2009.

[11] R. G. Vyas, "On the absolute convergence of small gaps Fourier series of functions of $\varphi \wedge B V$," JIPAM: Journal of Inequalities in Pure and Applied Mathematics, vol. 6, no. 4, article 94, 2005.

[12] M. Shiba, "On absolute convergence of Fourier series of function of class $\Lambda B V^{(p)}$," Fukushima University, no. 30, pp. 7-10, 1980.

[13] S. L. Wang, "Some properties of functions of $\Lambda$-bounded variation," Science in China, vol. 11, pp. 1299-1309, 1981 (Chinese).

[14] Y. E. Kuprikov, "On moduli of continuity of functions in Waterman classes," Moscow University Mathematics Bulletin, vol. 52, no. 5, pp. 46-49, 1997.

[15] U. Goginava, "On the embedding of Waterman class in the class $H_{p}^{\omega}$," Ukrainian Mathematical Journal, vol. 57, no. 11, pp. 18181824, 2005.

[16] U. Goginava, "Relations between $\Lambda B V$ and $B V(p(n) \uparrow \infty)$ classes of functions," Acta Mathematica Hungarica, vol. 101, no. 4, pp. 264-272, 2003.

[17] M. Hormozi, "Inclusion of $\Lambda B V^{(p)}$ spaces in the classes $H_{q}^{\omega}$," Journal of Mathematical Analysis and Applications, vol. 404, no. 2, pp. 195-200, 2013.

[18] M. Hormozi, A. A. Ledari, and F. Prus-Wiśniowski, "On $p-\Lambda$ bounded variation," Bulletin Iranian Mathematical Society, vol. 37, no. 4, pp. 35-49, 2011.

[19] O. Kováčik, "On the embedding $H^{\omega} \subset V_{p}$," Mathematica Slovaca, vol. 43, no. 5, pp. 573-578, 1993.

[20] L. Leindler, "On embedding of the class $H^{\omega}$," Journal of Inequalities in Pure and Applied Mathematics, vol. 5, no. 4, article 105, 2004.

[21] M. Lind, "On functions of bounded $\Lambda$-variation and integral smoothness," Forum Mathematicum, 2014.

[22] M. V. Medevedeva, "Embeddings of the class $H^{\omega}$," Mathematical Notes, vol. 64, no. 5, pp. 616-621, 1998.

[23] M. V. Medevedeva, "Conditions for embedding of classes $H^{\omega}$ into classes of functions of bounded generalized variation," Mathematical Bulletin, vol. 56, no. 2, pp. 41-45, 2001.

[24] M. V. Medvedeva, "Embeddings of classes of continuous functions into classes of functions of bounded generalized variation," Matematicheskiu Sbornik, vol. 193, no. 7, pp. 1049-1070, 2002.

[25] F. Prus-Wiśniowski, "On inclusion between Waterman classes and Chanturyia classes," Tatra Mountains Mathematical Publications, vol. 19, pp. 219-227, 2000. 


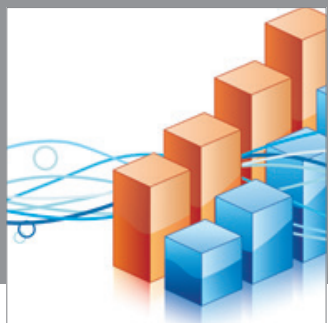

Advances in

Operations Research

mansans

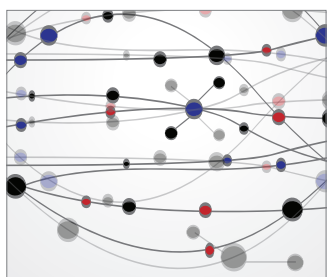

The Scientific World Journal
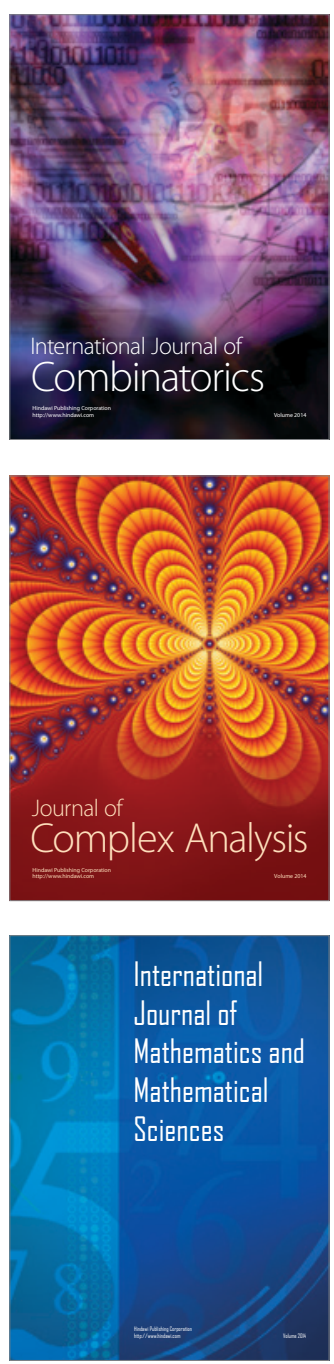
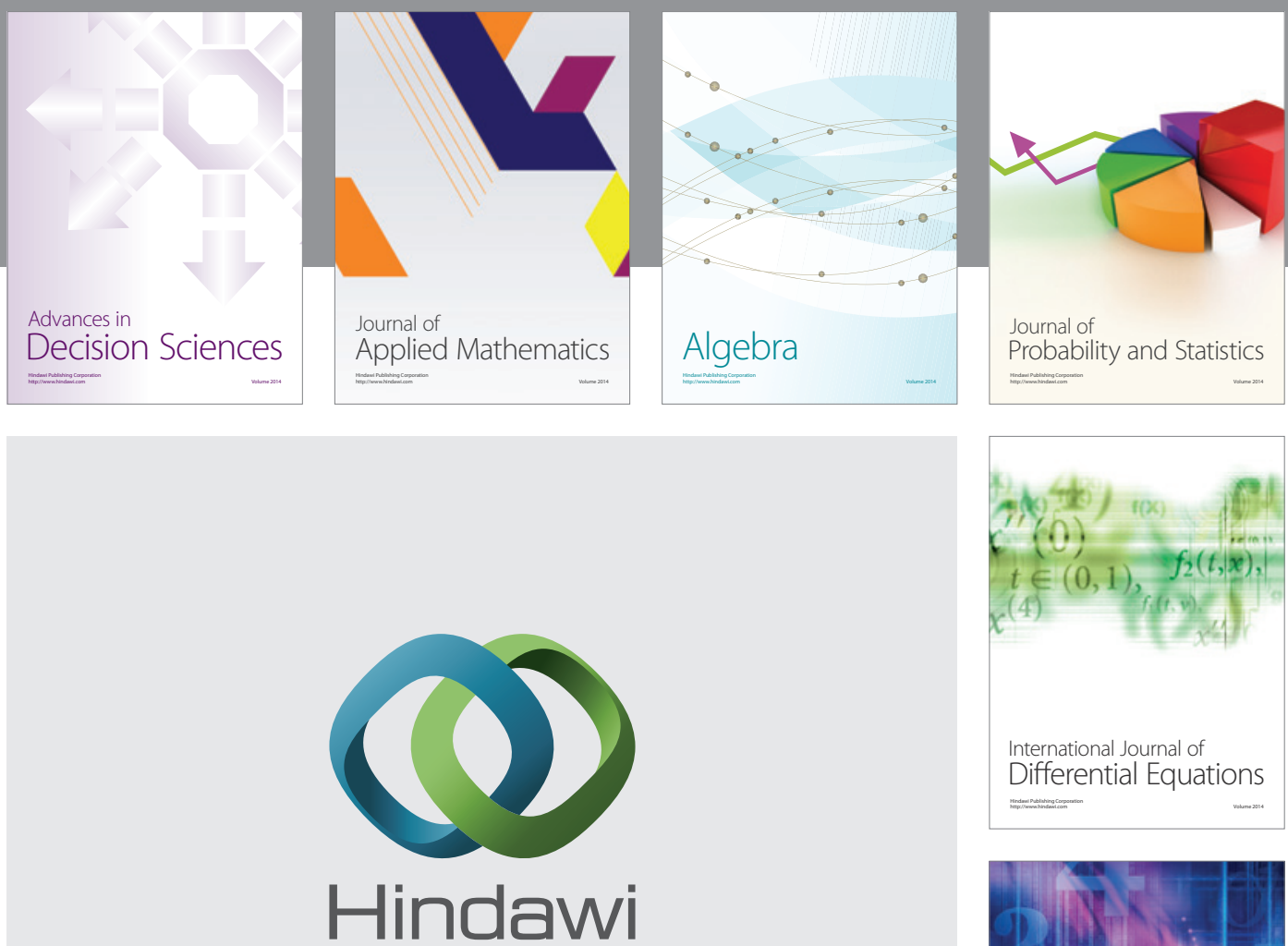

Submit your manuscripts at http://www.hindawi.com
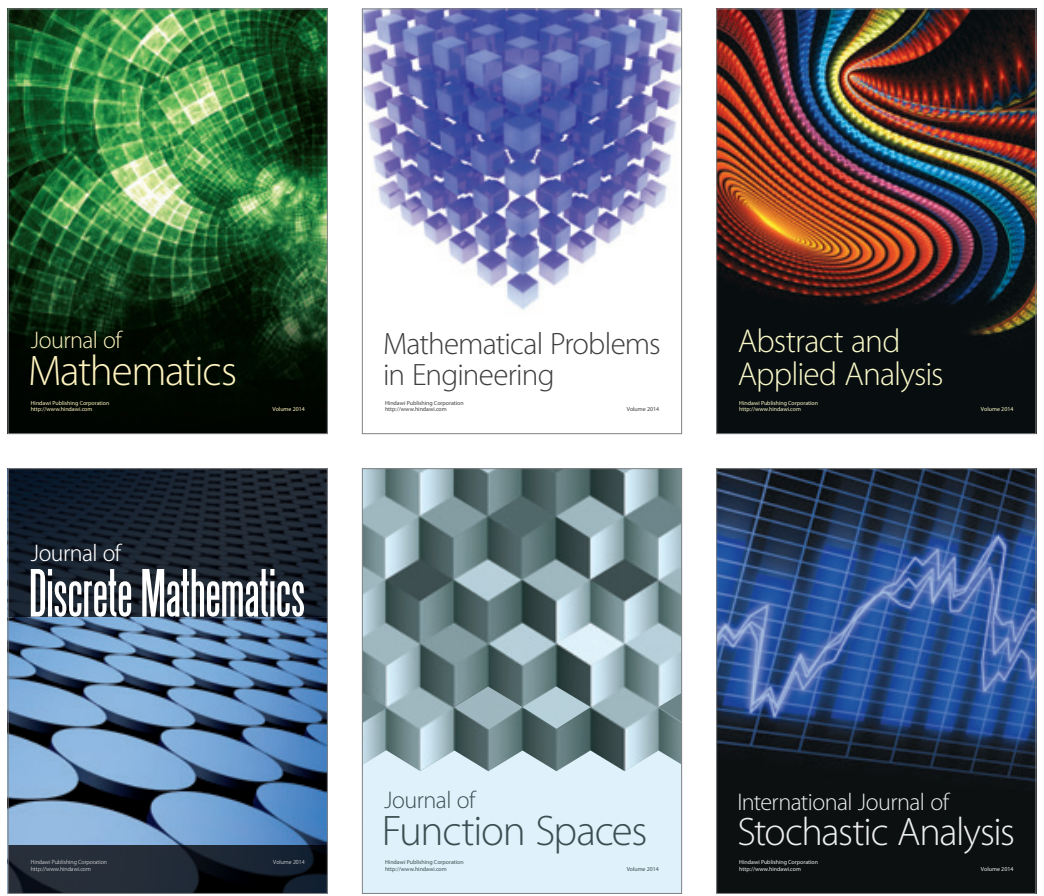

Journal of

Function Spaces

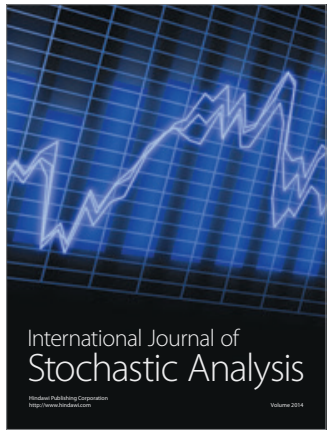

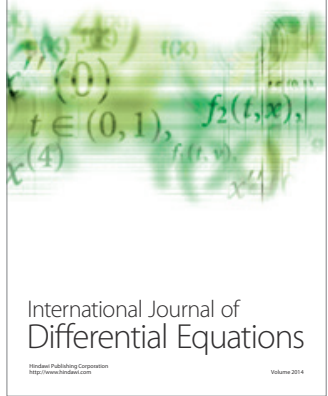
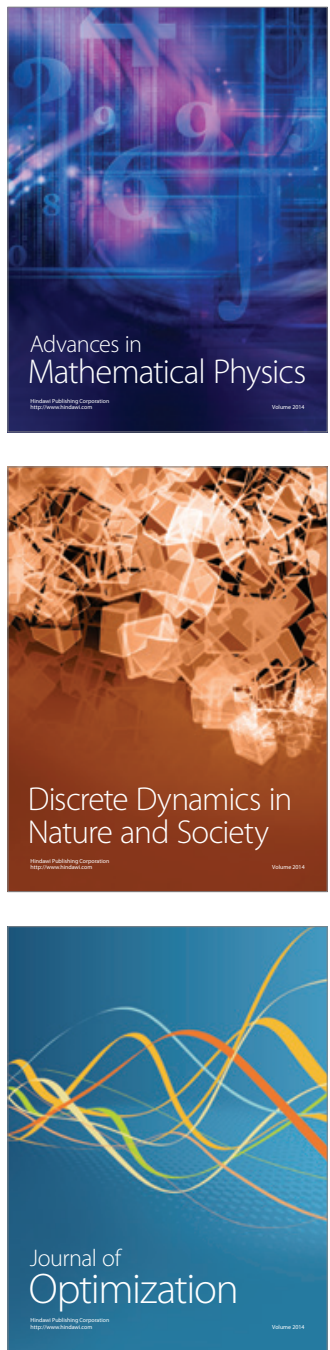